\title{
Using light and heat to controllably switch and reset disorder configuration in nanoscale devices
}

\author{
A. M. See, ${ }^{1}$ M. Aagesen, ${ }^{2}$ P. E. Lindelof, ${ }^{2}$ A. R. Hamilton, ${ }^{1}$ and A. P. Micolich ${ }^{1,}$ a \\ 1) School of Physics, University of New South Wales, Sydney NSW 2052, Australia \\ ${ }^{2)}$ Nanoscience center, University of Copenhagen, Universitetsparken 5, DK-2100 Copenhagen, \\ Denmark
}

(Dated: 7 July 2021)

\begin{abstract}
Quantum dots exhibit reproducible conductance fluctuations at low temperatures due to electron quantum interference. The sensitivity of these fluctuations to the underlying disorder potential has only recently been fully realized. We exploit this sensitivity to obtain a novel tool for better understanding the role that background impurities play in the electrical properties of high-mobility AlGaAs/GaAs heterostructures and nanoscale devices. In particular, we report the remarkable ability to first alter the disorder potential in an undoped AlGaAs/GaAs heterostructure by optical illumination and then reset it back to its initial configuration by room temperature thermal cycling in the dark. We attribute this behavior to a mixture of $\mathrm{C}$ background impurities acting as shallow acceptors and deep trapping by Si impurities. This 'alter and reset' capability, not possible in modulation-doped heterostructures, offers an exciting route to studying how scattering from even small densities of charged impurities influences the properties of nanoscale semiconductor devices.
\end{abstract}

Disorder is an important issue in nanoelectronics; as a device is reduced in size it becomes more sensitive to temporal fluctuations and spatial inhomogeneities in the charged impurity distribution. $\frac{1}{*}$ The temporal fluctuations cause decoherence ${ }^{2}, \underline{3}$, noise $e^{\underline{4}}$ and device irreproducibility $\underline{\underline{5} .6}$ These are troublesome for the development of quantum applications for semiconductor devices. Spatial inhomogeneities interrupt electron flow $\underline{7}$ adversely affecting ${ }^{8}$ practical realization of concepts such as topological quantum computation using the $5 / 2$ fractional quantum Hall state ${ }^{9}$. These barriers to applications have fueled research to reduce, control and better understand disorder in nanoscale electronic devices.

Approaches to disorder reduction for nanoscale devices include modulation-doping $\frac{10}{}$, short period superlattice doping ${ }^{11.12}$, and undoped heterostructures where the carriers are induced using either a metal ${ }^{13.14}$ or degenerately-doped semiconductor gate $e^{15,16}$. While modulation-doped structures still provide the highest mobilities, undoped heterostructures provide two distinct advantages. First, short range neutral disorder dominates over long range Coulombic disorder. This brings benefits such as enhanced robustness of the $5 / 2$ fractional quantum Hall state,,$\underline{8}$ and improved experimental access to the metallic state generated by electron-electron in-

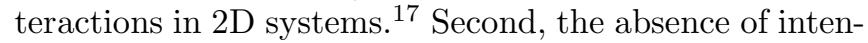
tional ionized impurities gives electrical properties that are remarkably robust to thermal cycling $\frac{18}{}$, in stark contrast to modulation-doped heterostructures ${ }^{6}$. Both features demonstrate there is much more to disorder than the popular metric of mobility alone $\stackrel{19}{\underline{1}}$ They also strongly motivate the quest for a deeper understanding of the nature of disorder in undoped heterostructure devices.
We recently developed a novel approach to studying disorder in nanoscale devices. It relies on the fact that a quantum dot's low temperature magnetoconductance shows quantum interference fluctuations that are highly sensitive to the paths electrons take in traversing the device ${ }^{20}$ These 'magnetoconductance fluctuations' (MCF) are influenced by both the dot geometry ${ }^{21}$ and the underlying disorder potential ${ }^{19,22}$ in dots smaller than the large-angle scattering length, i.e., in the 'ballistic' transport regime. If the dot geometry is fixed, changes in the MCF can be used to probe the physics of dopants, e.g., the intentional Si donors $\underline{6}$ and acceptors,$\underline{23}$ within the device. We recently made a remarkable finding - the MCF becomes highly reproducible, even after thermal cycling to room temperature, if intentional dopants are removed, i.e., the quantum dot is made using an undoped heterostructure $\underline{18}$ This result naturally leads to interesting questions: An undoped heterostructure is never $100 \%$ pure, so what role does the low density of non-intentional 'background' impurities inevitably incorporated into the heterostructure during growth play? Since these impurities are 'invisible' thermally, can we probe them another way, for example, optically? Might it be possible to use this small density of impurities as a new way to control electrical properties towards novel applications?

Here we demonstrate the ability to alter the disorder potential in undoped heterostructures by optical illumination, detect the resulting changes in spatial charge configuration by looking for changes in low temperature magnetoconductance, and then reset the disorder potential back to its initial configuration by thermally cycling the device to room temperature. This remarkable capability, not possible in conventional modulation-doped heterostructures, $\frac{6}{-}$ provides a significant opportunity to better understand how the spatial configuration of even small densities of charged impurities influences transport

\footnotetext{
a) Electronic mail: adam.micolich@nanoelectronics.physics.unsw.edu.au in nanoscale devices. Note that the technique could be
} 

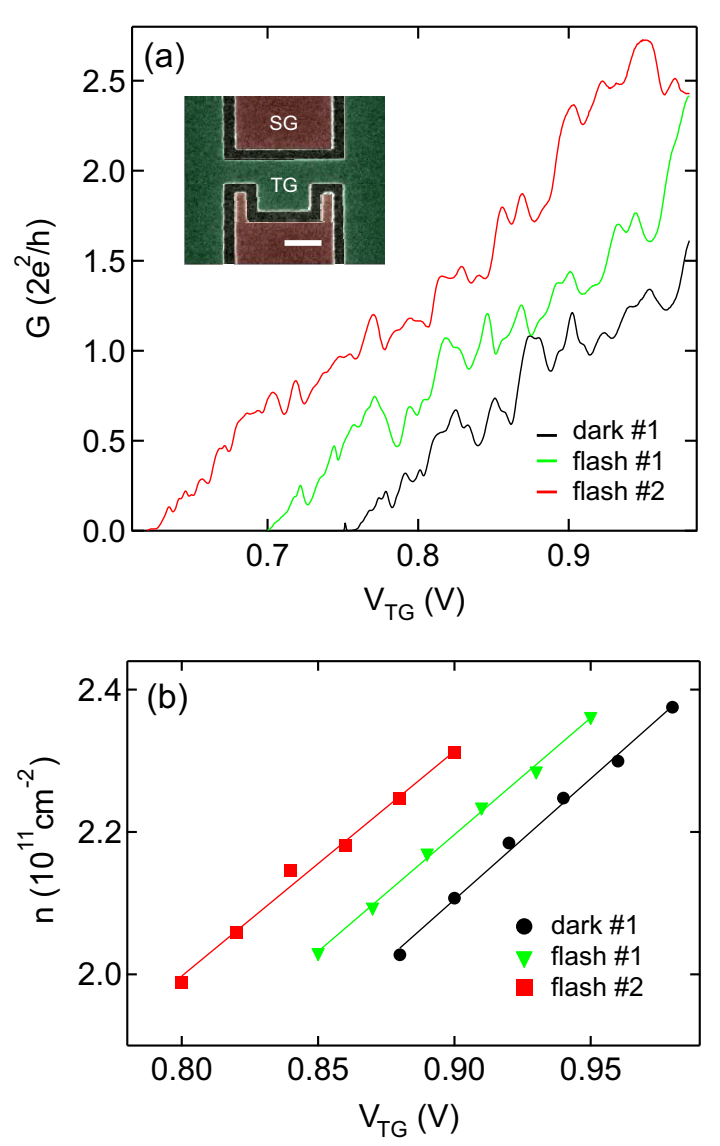

FIG. 1. The device and how the gate characteristics and density change with illumination. (a) A comparison of dot conductance $G$ vs top-gate voltage $V_{T G}$ obtained at temperature $T=250 \mathrm{mK}$ and side-gate voltage $V_{S G}=0 \mathrm{~V}$. The three traces shown are: (black) prior to illumination denoted 'dark \#1', (green) after first illumination denoted 'flash \#1', and (red) after second illumination denoted 'flash \#2'. (Inset) Scanning electron micrograph of the device; the scale bar represents $500 \mathrm{~nm}$. The top-gate (TG) is shaded green, the side-gates (SG) are shaded red. (b) Two-dimensional electron density $n$ vs. $V_{T G}$ for different amounts of illumination. The straight lines are fits to the experimental data.

readily extended towards materials beyond III-V semiconductors, e.g., graphene, $\stackrel{24,25}{\stackrel{2 n}{2}}$ one only needs a quantum dot showing quantum interference fluctuations.

A scanning electron micrograph of our device is shown inset to Fig. 1(a). It was made using a heterostructure consisting of an undoped GaAs substrate overgrown with $160 \mathrm{~nm}$ undoped AlGaAs, $25 \mathrm{~nm}$ undoped GaAs, and a $35 \mathrm{~nm} \mathrm{n}^{+}$GaAs cap, which remains highly conductive at low temperature. The cap is divided into three independent gates - a top-gate (green) and two side-gates (red) - using electron-beam lithography and shallow wet etching. The NiGeAu ohmic contacts are produced using an established self-aligned process $\underline{16,26}$ The dot and the $2 \mathrm{D}$ electron gas (2DEG) source and drain reservoirs are populated electrostatically when the top-gate (TG) is biased to a sufficiently positive voltage $V_{T G}$. The width of the quantum point contacts (QPCs) connecting the dot to source and drain can be tuned via a negative voltage $V_{S G}$ applied to the side-gates (SG). Electrical characterization of a Hall bar made using the same heterostructure at $4.2 \mathrm{~K}$ gave a mobility $\mu \sim 300,000 \mathrm{~cm}^{2} / \mathrm{Vs}$ at $n \sim 1.8 \times 10^{11} \mathrm{~cm}^{-2}$, corresponding to $\ell \sim 2.1 \mu \mathrm{m}$, which is larger than the dot dimensions $(760 \times 660 \mathrm{~nm})$. Electrical measurements were performed at temperature $T \approx 250 \mathrm{mK}$ using a pumped ${ }^{3} \mathrm{He}$ cryostat (Oxford Instruments Heliox), with the magnetoconductance $G(B)$ obtained by standard four-terminal lock-in techniques using a fixed $100 \mu \mathrm{V}$ excitation voltage at a frequency of $11 \mathrm{~Hz}$. The variable magnetic field $B$ was applied along the heterostructure growth direction (perpendicular to the plane of Fig. 1(a) inset). Illumination was performed using a red LED (see Fig. S1 for emission spectrum) mounted in close proximity to the device. Illumination is implemented via a control box that drives an LED current of $1 \mathrm{~mA}$ for a single $100 \mathrm{~ms}$ pulse. Further details on the undoped devices and electrical measurements are available elsewhere $\underline{16,18,26}$

Figure 1(a) shows traces of zero field conductance $G(B=0)$ versus $V_{T G}$ obtained in a single cooldown: before illumination denoted 'dark \#1' (red), after a $100 \mathrm{~ms}$ illumination denoted 'flash \#1' (black), and after another $100 \mathrm{~ms}$ illumination denoted 'flash \#2' (green). In each case $G$ increases as $V_{T G}$ is made more positive, reflecting a gate-induced accumulation of electrons in the dot and source/drain contacts. The threshold voltage $V_{t h}-$ defined here as the lowest $V_{T G}$ where $G$ becomes non-zero - decreases from $0.76 \mathrm{~V}$ to $0.7 \mathrm{~V}$ and then $0.62 \mathrm{~V}$ after the first and second illuminations, respectively. This $V_{t h}$ shift indicates the optical ionization of background impurities and a commensurate increase in electron density. To more clearly demonstrate the density increase in Fig. 1(b) we plot the electron density $n$ from Shubnikovde Haas measurements of an adjacent Hall-bar segment on this device versus $V_{T G}$; the red circles, black triangles and green squares are data obtained before, between and after the two illuminations, respectively. With each illumination, the density increases by $4-5 \%$. This density increase is persistent, as previously shown in an inverted, undoped AlGaAs/GaAs heterointerface ${ }^{27}$ The slope of $n$ versus $V_{T G}$ in Fig. 1(b) is constant, demonstrating that illumination does not alter the gate-2DEG capacitance.

The fluctuations in the gate sweeps in Fig. 1(a) arises from quantum interference ${ }^{28}$ and represents a 'fingerprint' of transport through the dot similar to $G(B)$. The exact fluctuation pattern varies with each illumination due to a change in the disorder potential within the dot. We can assess this further using $G(B), \stackrel{6,18}{ }$ but doing this rigorously requires caution. If we simply compare $G(B)$ at a given $V_{T G}$ before, between and after illuminations, the disorder potential change is masked by an associated density-driven change in Fermi wavelength $\lambda_{F} \sim n^{-\frac{1}{2}}$ (for completeness, we nonetheless present this comparison in Fig. S2); such a comparison is only meaningful at common $n$. To properly demonstrate the illumination- 
induced change in disorder potential we use the following procedure. First, we plot the gate sweeps to a transformed gate voltage axis $V_{T G}^{\prime}=V_{T G}-V_{t h}$ as shown in Fig. 2(a). The three traces have a common gradient but different fluctuation patterns such that the traces cross each other at various points. At these crossing points the device has the same $G(B=0)$ and $n$ for a given $V_{T G}^{\prime}$ before, between and after the two illuminations, providing the most fair basis for comparing the corresponding $G(B)$ traces. One such instance is highlighted by the arrow at $V_{T G}^{\prime}=+0.90 \mathrm{~V}$ in Fig. 2(a) inset; the three corresponding $G(B)$ traces are plotted in Fig. 2(b). The $G(B)$ traces vary significantly confirming a change in the dot's disorder potential. $\underline{6,18}$ This is highlighted in Fig. 2(c), where we plot the difference $\delta G=$ $G(B)_{\text {flash \#1 }}-G(B)_{\text {dark \#1 }}$ (solid), $G(B)_{\text {flash \#2 }}-$ $G(B)_{\text {flash \#1 }}$ (dashed), or $G(B)_{\text {flash \#2 }}-G(B)_{\text {dark } \# 1}$ (dotted) versus $B$. The latter highlights the cumulative change arising from the two illuminations. The cumulative rms fluctuation across the $0<B<0.5 \mathrm{~T}$ range is $0.076,0.088$ and $0.108 \times 2 e^{2} / h$, respectively. Each illumination causes an approximately equivalent change, but this effect ultimately diminishes due to the finite population of light-active centers; this typically occurs after four $100 \mathrm{~ms}$ illumination pulses for this device.

The most remarkable aspect of this device emerges in response to thermal cycling to room temperature. Figure $3(\mathrm{a})$ shows $G(B=0)$ versus $V_{T G}$ from the second cooldown before illumination, denoted dark \#2 (solid), along with the corresponding trace from the first cooldown dark \#1 (dashed). These two traces are essentially identical, as are $G(B)$ traces obtained at $V_{T G}=$ $+0.9 \mathrm{~V}$ (Fig. 3(b)). Note that $V_{T G}=+0.9 \mathrm{~V}$ is chosen out of convenience here; matching $G(B)$ for various $V_{T G}$ as reported previously 18 The $G(B)$ similarity is clear in the difference trace $\delta G(B)=G(B)_{\text {dark } \# 2}-G(B)_{\text {dark }} \# 1$ in Fig. 3(c), which is presented at matching scale to Fig. 2(c) for direct comparison. The cumulative rms fluctuation for the trace in Fig. 3(c) is $0.030 \times 2 e^{2} / h$, a factor of $2-4$ smaller than for the traces in Fig. 2(c). This indicates that the disorder potential returns to its initial configuration upon thermal cycling. In other words, in our undoped devices, illumination can be used to randomly reconfigure the disorder, and it can be 'reset' to a repeatable base configuration by thermal cycling.

An interesting question is: What is the nature of the disorder enabling this ability to optically alter and then thermally reset the disorder potential? Two features of the data provide important clues. The first is that illumination increases the electron density (Fig. 1(b)), i.e., the sample displays persistent positive photoconductivity $\underline{29}-\underline{31}$ The second is that the impurity population returns to its initial ionization configuration after thermal cycling (Fig. 3(a/b)). There are two possible impurity models that satisfy these features: Si background impurities acting as deep traps and $\mathrm{C}$ background impurities acting as shallow acceptors; both may exist at different densities. We first address these individu-
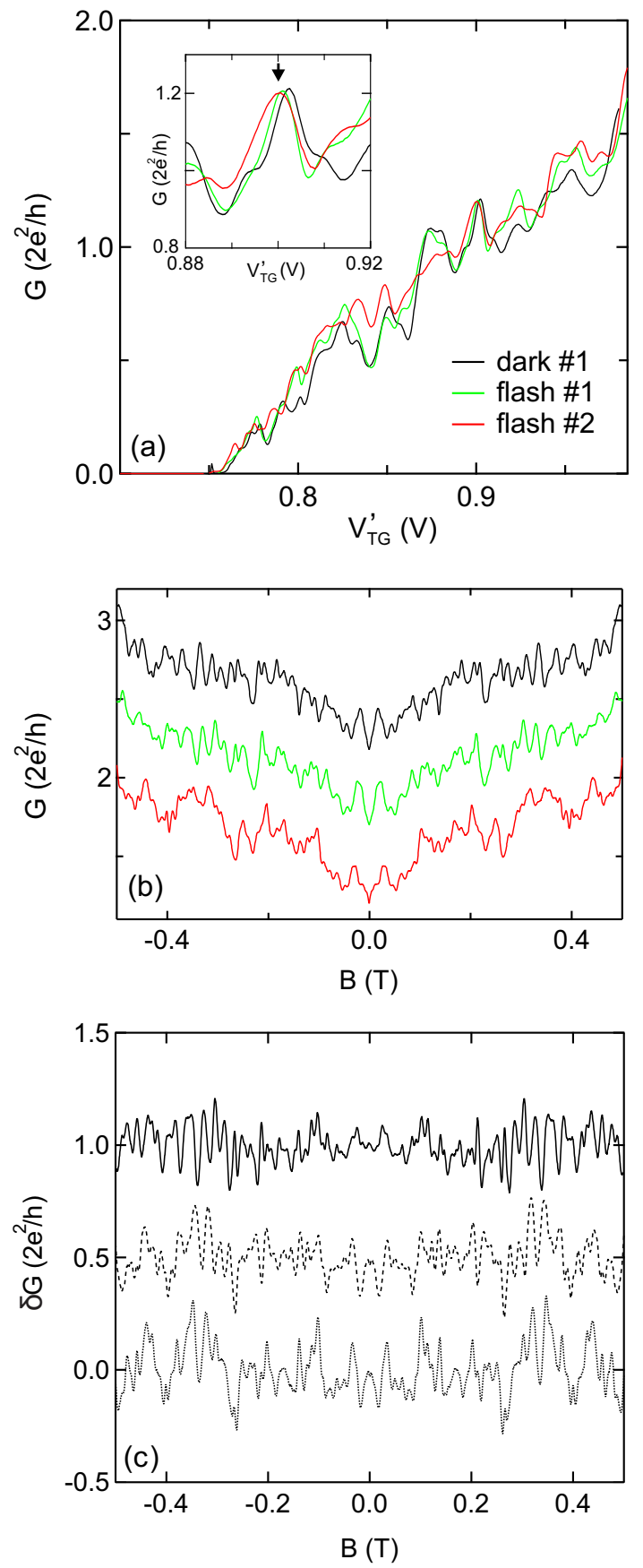

FIG. 2. Demonstration that illumination changes the dot's disorder potential. (a) Comparison of $G$ vs transformed top gate voltage $V_{T G}^{\prime}=V_{T G}-V_{t h}$ where $V_{t h}=0,55,130 \mathrm{mV}$ for the dark \#1 (black), flash \#1 (green) and flash \#2 (red) traces, respectively, using data from Fig. 1(a). (Inset) A magnified view of the main panel centered at $V_{T G}^{\prime}=0.90 \mathrm{~V}$ showing a crossing point for the three traces as indicated by the arrow. (b) Dark \#1, flash \#1 and flash \#2 $G$ vs $B$ traces obtained at $V_{T G}=0.900 \mathrm{~V}$ (black), $0.845 \mathrm{~V}$ (green) and $0.770 \mathrm{~V}$ (red) corresponding to $V_{T G}^{\prime}=0.90 \mathrm{~V}$. (c) The MCF difference $\delta G=G(B)_{\text {flash \#1 }}-G(B)_{\text {dark \#1 }}$ (solid), $G(B)_{\text {flash \#2 }}-$

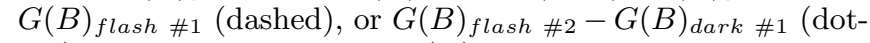
ted) vs $B$ highlighting the $G(B)$ changes resulting from illumination. Traces in (b) and (c) are offset sequentially downwards by $0.5 \times 2 e^{2} / h$ for clarity. 
ally with respect to persistent photoconductivity, before considering how they fit with our observation that we can reset the MCF, and thereby the disorder potential, to its initial configuration by room temperature thermal cycling.

A well known cause of persistent positive photoconductivity in $\mathrm{Al}_{x} \mathrm{Ga}_{1-x} \mathrm{As} / \mathrm{GaAs}$ heterostructures with $x>0.2$ are deep-trapping Si DX centers in the AlGaAs layer ${ }^{32.33}$ Briefly, the Si DX center can take three possible states: shallow hydrogenic donor states $\mathrm{d}^{0}$ and $\mathrm{d}^{+}$, and a deep trap $\mathrm{DX}^{-}$that is stabilized by lattice deformation ${ }^{32,34}$ The ground-state is $\mathrm{DX}^{-}$, it can be optically excited to release one or both of its trapped electrons to become $\mathrm{d}^{0}$ or $\mathrm{d}^{+}, 34,35$ The latter is the ultimate outcome either way, as the transition $\mathrm{d}^{0} \rightarrow \mathrm{d}^{+}$ requires much less energy than $\mathrm{DX}^{-} \rightarrow \mathrm{d}^{0} .35$ The optically liberated electrons either join the 2DEG or are swept into the $\mathrm{n}^{+}$cap by the gate electric field. Note that these liberated electrons cannot be retrapped by $\mathrm{Si}$ impurities unless the sample is warmed above $100 \mathrm{~K}$ due to a lattice-deformation-induced energy barrier surrounding these impurities. 33 Thus an associated change in the charge between gate and 2DEG occurs, producing a negative shift in $V_{t h}$, and equivalently, an increase in $n$ at fixed $V_{T G}$, i.e., persistent photoconductivity. The presence of Si background impurities in our device are a logical possibility because a) the $2 \mathrm{DEG}$ is immediately adjacent to a $160 \mathrm{~nm}$ undoped $\mathrm{Al}_{0.33} \mathrm{Ga}_{0.67}$ As layer and b) the MBE chamber contains a $\mathrm{Si}$ source for degenerate doping of the GaAs cap in these structures and modulation-doping of other heterostructures grown during the same chamber evacuation. Ge, S, Se, Sn and Te impurities can also produce deep-trapping DX centers in AlGaAs; $; 33,36$ in particular, $\mathrm{S}$ is a common impurity in As MBE sources 37,38 and is proposed to also show DX behavior in GaAs $\stackrel{39,40}{ }$ Note that $\mathrm{Si}$ is a shallow hydrogenic donor in GaAs with states $\mathrm{d}^{0}$ and $\mathrm{d}^{+}$only.

$\mathrm{C}$ background impurities are also common in MBEgrown AlGaAs/GaAs heterostructures ${ }^{37.38}$ Carbon is not known to show DX behavior, 33 acting instead as a shallow acceptor $\stackrel{41}{ }$ However, an ionized $\mathrm{C}$ acceptor $\mathrm{a}^{-}$ may be neutralized to $\mathrm{a}^{0}$ by hole-capture from a nearby photo-generated electron-hole pair. This electron-hole pair generation should occur, particularly in our GaAs layers, since we use a red LED in this experiment. 42 This process should result in positive persistent photoconductivity providing the photo-generated electron enters the 2DEG or $\mathrm{n}^{+}$-GaAs cap, rather than being captured to reionize the same acceptor. This is possible because, unlike the DX center, there is no lattice-deformation-induced barrier surrounding the $\mathrm{C}$ impurity. Illumination can decrease the two-dimensional hole gas density in C-doped AlGaAs/GaAs heterostructures, ${ }^{43}$ presumably via a similar mechanism. One advantage of this model is that it is viable for both the undoped AlGaAs spacer and the GaAs substrate, unlike the Si DX center model discussed above.

We now turn our attention to the ability to reset
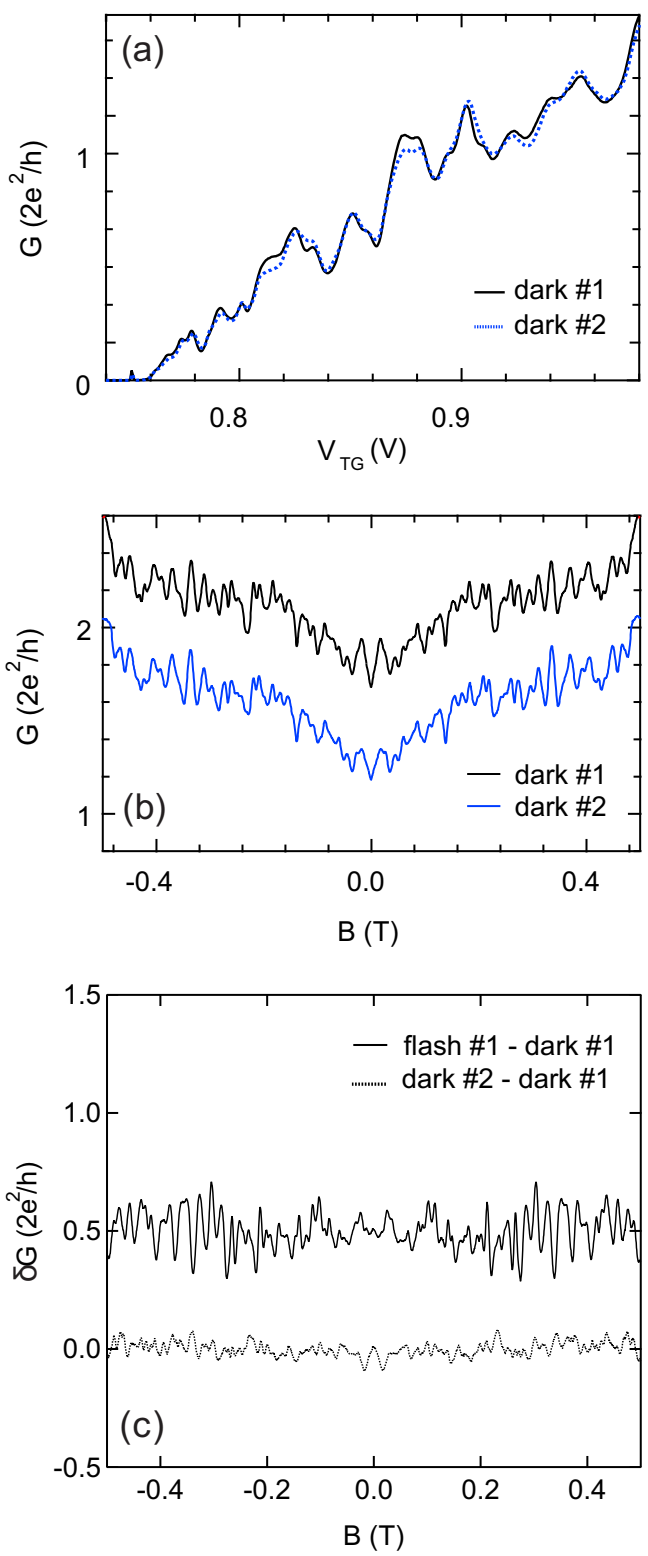

FIG. 3. Demonstration of the ability to reset the disorder potential to its initial configuration with thermal cycling. (a) Comparison of $G$ vs $V_{T G}$ obtained before illumination on the first cooldown, denoted dark \#1 (black solid line), and after two illuminations and room temperature thermal cycling in the dark, denoted dark \#2 (blue dashed line). (b) Comparison of $G$ vs. $B$ at $V_{T G}=0.9 \mathrm{~V}$ for dark $\# 1$ (black) and dark \#2 (blue); the dark \#2 trace is offset vertically by $0.5 \times 2 e^{2} / h$ for clarity. The red trace is offset upwards by $0.5 \times 2 e^{2} / h$ for clarity. (c) The MCF differ-

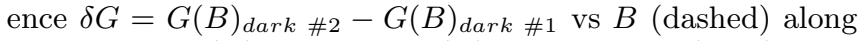

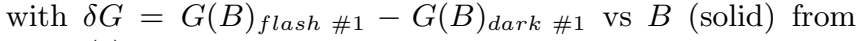
Fig. 2(c) for comparison. The solid trace is offset upwards by $0.5 \times 2 e^{2} / h$ for clarity. 
the disorder potential and MCF to the initial, preillumination configuration by room temperature thermal cycling. The fact that we observe this means that upon cool-down all impurities return to their initial charge states. In the simplest case, this implies: a) all acceptors, e.g., C impurities, are in the ionized $\mathrm{a}^{-}$state, b) all donors, e.g., $\mathrm{Si}$, S, etc. in GaAs are in the $\mathrm{d}^{+}$state, and c) all donors, e.g., Si, S, etc. in the AlGaAs are in the $\mathrm{DX}^{-}$state. While this ignores some exotic possibilities, e.g., impurity complexes, these are likely rare due to the very low impurity density. We now consider the plausibility of this full ionization scenario. As a shallow acceptor, $\mathrm{C}$ in GaAs commonly shows electrical activation efficiencies well above $80 \% \underline{\text {, }}$, with $100 \%$ activation found for doping densities less than $3 \times 10^{17} \mathrm{~cm}^{-3} \underline{\underline{44} \mathrm{Ac}-}$ tivation is enhanced in $\mathrm{Al}_{x} \mathrm{Ga}_{1-x} \mathrm{As}$, increasing with $\mathrm{Al}$ content $x: \underline{41}$ We also expect full ionization of Si shallow donors in GaAs as their activation energy is $6 \mathrm{meV}$, compared to $26 \mathrm{meV}$ for $\mathrm{C}$ shallow acceptors $\underline{45}$ Complete $\mathrm{Si}$ activation is indeed observed at low doping densities $;^{46}$ autocompensation due to Si dopant amphotericity $\underline{47}$ and more complex donor deactivation mechanisms $\frac{48}{}$ only become important for Si densities above $10^{19} \mathrm{~cm}^{-3}$. The return of all Si impurities in the AlGaAs to the $\mathrm{DX}^{-}$state is the most unexpected outcome because in modulationdoped devices this does not occur $\underline{6,34}$ However, the intentional Si density in modulation-doped structures is $1,000-2,000 \times$ greater than the background impurity density in these undoped heterostructures,$\frac{49}{,}$ even if we assume that all of the background impurities are $\mathrm{Si}$, which is highly unlikely. In this instance, the free electron density in the AlGaAs during cool-down may sufficiently exceed the Si impurity density to doubly-occupy all DX centers - there may be two coinciding mechanisms that aid this process. The first is the tendency for background impurities, particularly $\mathrm{Si}$, to ride upwards on the AlGaAs growth front 50,51 This would push the bulk of the Si background impurities closer to the $\mathrm{n}^{+}$-GaAs cap. The second is that the Fermi energy is pinned against the conduction band edge in the $\mathrm{n}^{+}$-GaAs cap, which raises the local Fermi level in the upper parts of the AlGaAs spacer (e.g. see Fig. 2 of Ref. ${ }^{52}$ ). This should enhance the free electron density in the AlGaAs layer, increasing the probability for Si background impurities to take the $\mathrm{DX}^{-}$state. Additionally, the lower Si background impurity density would increase the impurity spacing by a factor of at least 10 relative to modulation-doped heterostructures. This large spacing $\gtrsim 65 \mathrm{~nm}$ should dramatically reduce charge migration between adjacent DX centers (i.e., $\mathrm{DX}^{-}+\mathrm{d}^{+} \rightarrow \mathrm{d}^{+}+\mathrm{DX}^{-}$), further enhancing MCF stability. It should also prevent charge migration between acceptor sites, and the associated slow drift in $G(B)$ recently reported for hole quantum dots ${ }^{23}$

Turning to the mobility, the transition $\mathrm{a}^{-} \rightarrow \mathrm{a}^{0}$ should result in a mobility increase as it eliminates a charged scattering center. In contrast, there should be little change in the mobility from the transition $\mathrm{DX}^{-} \rightarrow \mathrm{d}^{+}$because negative $\mathrm{DX}^{-}$centers and positive $\mathrm{d}^{+}$donors both
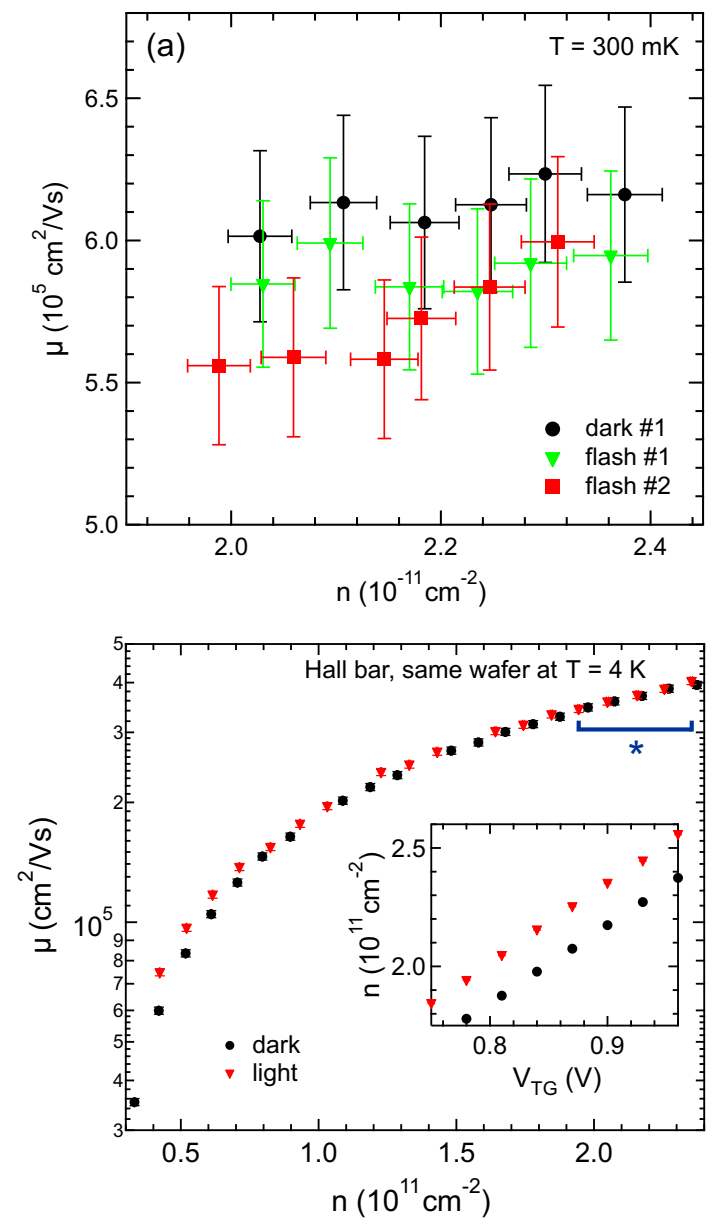

FIG. 4. How illumination influences the mobility (a) The mobility $\mu$ vs electron density $n$ for dark \#1 (black circles), flash \#1 (green triangles) and flash \#2 (red squares) measured from Shubnikov-de Haas oscillations measured on a Hall-bar segment immediately adjacent to the quantum dot used to obtain data in Figs. 1-3. Data was obtained at $T=300 \mathrm{mK}$ from a region of the Hall-bar adjacent to the quantum dot. (b) The mobility $\mu$ vs electron density $n$ obtained before (black circles) and after illumination (red triangles) measured for a separate Hall-bar sample made using the same heterostructure. Only the error bars in $\mu 2 \%$ are shown, the error bars in $n 0.2 \%$. The data was obtained at $T=4 \mathrm{~K}$ and the blue bracket marked $*$ indicates the operating density range for our quantum dot sample (cf. Fig. 1(b)). Inset shows $n$ vs top-gate voltage $V_{T G}$ for this Hall bar for comparison with data from the quantum dot sample in Fig. 1(b).

cause Coulomb scattering of electrons in the 2DEG. This argument ignores DX center correlation effects,,$\frac{34}{4}$ which might be justified given the $\sim 10 \times$ higher donor separation; but for completeness, were there any correlations the random nature of DX center photoionization should reduce them, causing $\mu$ to decrease slightly ${ }^{34}$ Figure 4(a) shows $\mu$ vs $n$ obtained Shubnikov-de Haas measurements from a Hall bar segment immediately adjacent to the quantum dot used for Figs. 1-3. This data suggests that $\mu(n)$ decreases with illumination, but the trend is barely 
significant relative to measurement error. In Fig. 4(b) we present characterization data for a separate Hall bar from the same heterostructure without any etched quantum dot. Here we observe an increase in $\mu(n)$ with illumination, which ranges from over $20 \%$ at low $n$ to almost zero at high $n>2 \times 10^{15} \mathrm{~cm}^{-2}$ where our device is operated (indicated by the blue $*$ in Fig. 4(b)). A similar increase in $\mu(n)$ was previously reported for undoped AlGaAs/GaAs heterostructures by Saku et al. $\stackrel{27}{ }$, but their increase was much larger, decreasing from $\sim 46 \%$ at $n \sim 2 \times 10^{10} \mathrm{~cm}^{-2}$ to $\sim 25 \%$ at $n \sim 2 \times 10^{11} \mathrm{~cm}^{-2}$. Given the lack of a clear shift in $\mu$ with illumination in our operating $n$ range, we suspect that both mechanisms $\mathrm{a}^{-} \rightarrow \mathrm{a}^{0}$ and $\mathrm{DX}^{-} \rightarrow \mathrm{d}^{+}$are at play in our device, perhaps with the $\mathrm{a}^{-} \rightarrow \mathrm{a}^{0}$ process being slightly dominant. Additional studies using custom doped heterostructures and/or allied analytical methods, e.g. deep level transient spectroscopy (DLTS) $\lcm{55}$ may assist further in understanding the physics of the underlying dopant photoexcitation processes involved in the device behavior we report.

In summary, we have shown the ability to alter the disorder potential in undoped heterostructures by optical illumination, and then reset the disorder potential back to its initial configuration by thermally cycling the device to room temperature. Our data suggests that this process likely arises from a mixture of two processes: a) photoexcitation of ionized $\mathrm{C}$ background acceptors back to their neutral state, and b) photoionization of Si DX centers. This remarkable 'alter and reset' capability is not possible in conventional modulation-doped heterostructures 6 and offers an exciting new route to studying how spatial aspects of scattering from even small densities of charged impurities influences transport in nanoscale semiconductor devices. Our approach also has great potential as a tool for optimising semiconductor materials for applications where extremely low disorder/scattering is vital to operation, e.g., quantum information processing, $, 8,12$ including materials beyond III-V semiconductors, e.g., graphene. 24,25

This work was funded by the Australian Research Council (ARC). APM acknowledges an ARC Future Fellowship and ARH acknowledges an ARC Outstanding Researcher Award. We thank L. Eaves, D.J. Carrad and S.P. Bremner for helpful discussions. This work was performed in part using the NSW node of the Australian National Fabrication Facility (ANFF).

${ }^{1}$ M.Y. Simmons, Nature Physics 4, 165 (2008).

${ }^{2}$ X. Hu, and S. Das Sarma, Phys. Rev. Lett. 96, 100501 (2006).

${ }^{3}$ K.D. Petersson, J.R. Petta, H. Lu and A.C. Gossard, Phys. Rev. Lett. 105, 246804 (2010).

${ }^{4}$ C. Kurdak, C.J. Chen, D.C. Tsui, S. Parihar, S. Lyon and G.W. Weimann, Phys. Rev. B 56, 9813-9818 (1997).

${ }^{5}$ Q.-Z. Yang, M.J. Kelly, I. Farrer, H.E. Beere and G.A.C. Jones, Appl. Phys. Lett. 94, 033502 (2009).

${ }^{6}$ B.C. Scannell, I. Pilgrim, A.M. See, R.D. Montgomery, P.K. Morse, M.S. Fairbanks, C.A. Marlow, H. Linke, I. Farrer, D.A. Ritchie, A.R. Hamilton, A.P. Micolich, L. Eaves and R.P. Taylor, Phys. Rev. B 85, 195319 (2012).
${ }^{7}$ M.P. Jura, M.A. Topinka, L. Urban, A. Yazdani, H. Shtrikman, L.N. Pfeiffer, K.W. West and D. Goldhaber-Gordon, Nature Physics 3, 841-845 (2007).

${ }^{8}$ W. Pan, N. Masuhara, N.S. Sullivan, K.W. Baldwin, K.W. West, L.N. Pfeiffer and D.C. Tsui, Phys. Rev. Lett. 106, 206806 (2011). ${ }^{9}$ C. Nayak, S.H. Simon, A. Stern, M. Freedman and S. Das Sarma, Rev. Mod. Phys. 80, 1083-1159 (2008).

${ }^{10}$ R. Dingle, H.L. Störmer, A.C. Gossard and W. Wiegmann, Appl. Phys. Lett. 33, 665-667 (1978).

${ }^{11}$ K.-J. Friedland, R. Hey, H. Kostial, R. Klann and K. Ploog, Phys. Rev. Lett. 77, 4616-4619 (1996).

${ }^{12}$ V. Umansky, M. Heiblum, Y. Levinson and J. Smet, J. Cryst. Growth 311, 1658-1661 (2009).

${ }^{13}$ Y. Hirayama, J. Appl. Phys. 80, 588-590 (1996).

${ }^{14}$ R.H. Harrell, K.S. Pyshkin, M.Y. Simmons, D.A. Ritchie, C.J.B. Ford, G.A.C. Jones and M. Pepper, Appl. Phys. Lett. 74, 23282330 (1999).

${ }^{15}$ P.M. Solomon, C.M. Knoedler and S.L. Wright, IEEE Electron Device Lett. 5, 379-381 (1984).

${ }^{16}$ B.E. Kane, L.N. Pfeiffer, K.W. West and C.K. Harnett, Appl. Phys. Lett. 63, 2132-2134 (1993).

${ }^{17}$ W.R. Clarke, C.E. Yasin, A.R. Hamilton, A.P. Micolich, M.Y. Simmons, K. Muraki, Y. Hirayama, M. Pepper and D.A. Ritchie, Nat. Phys. 4, 55-59 (2008).

${ }^{18}$ A.M. See, I. Pilgrim, B.C. Scannell, R.D. Montgomery, O. Klochan, A.M. Burke, M. Aagesen, P.E. Lindelof, I. Farrer, D.A. Ritchie, R.P. Taylor, A.R. Hamilton and A.P. Micolich, Phys. Rev. Lett. 108, 196807 (2012).

${ }^{19}$ A.P. Micolich, A.M. See, B.C. Scannell, C.A. Marlow, T.P. Martin, I. Pilgrim, A.R. Hamilton, H. Linke and R.P. Taylor, Fortschr. Phys. 61, 332-347 (2013).

${ }^{20}$ S. Feng, P.A. Lee and A.D. Stone, Phys. Rev. Lett. 56 1960-1963 (1986).

${ }^{21}$ C.M. Marcus, A.J. Rimberg, R.M. Westervelt, P.F. Hopkins and A.C. Gossard, Phys. Rev. Lett. 69, 506-509 (1992).

${ }^{22}$ Z.-L. J, and K.-F. Berggren, Phys. Rev. B 52, R11607-R11610 (1995).

${ }^{23}$ D.J. Carrad, A.M. Burke, O. Klochan, A.M. See, A.R. Hamilton, A. Rai, D. Reuter, A.D. Wieck and A.P. Micolich, Phys. Rev. B 89, 155313 (2014).

${ }^{24}$ N.E. Staley, C.P. Puls and Y. Liu, Phys. Rev. B 77, 155429 (2008).

${ }^{25}$ Y. Ujiie, S. Motooka, t. Morimoto, N. Aoki, D.K. Ferry, J.P. Bird and Y. Ochiai, J. Phys.: Condens. Matter 21, 382202 (2009).

${ }^{26}$ A.M. See, O. Klochan, A.R. Hamilton, A.P. Micolich, M. Aagesen and P.E. Lindelof, Appl. Phys. Lett. 96, 112104 (2010).

${ }^{27}$ T. Saku, K. Muraki and Y. Hirayama, Jpn. J. Appl. Phys. 37, L765-L767 (1998).

${ }^{28}$ J.P. Bird, R. Akis, D.K. Ferry, D. Vasileska, J. Cooper, Y. Aoyagi and T. Sugano, Phys. Rev. Lett. 82, 4691 (1999).

${ }^{29}$ H.L. Störmer, A.C. Gossard, W. iegmann and K. Baldwin, Appl. Phys. Lett. 39, 912-914 (1981).

${ }^{30}$ D.V Lang and R.A. Logan, Phys. Rev. Lett. 39, 635-639 (1977).

${ }^{31}$ R.J. Nelson, Appl. Phys. Lett. 31, 351-353 (1977).

32 P.M. Mooney, J. Appl. Phys. 67, R1-R26 (1990).

${ }^{33}$ D.V. Lang, R.A. Logan and M. Jaros, Phys. Rev. B 19, 10151030 (1979).

${ }^{34}$ E. Buks, M. Heiblum, Y. Levinson and H. Shtrikman, Semicond. Sci. Technol. 9, 2031-2041 (1994).

${ }^{35}$ P.M. Mooney, Semicond. Sci. Technol. 6, B1-B8 (1991).

${ }^{36}$ O. Kumagai, H. Kawai, Y. Mori and K. Kaneko, Appl. Phys. Lett. 45, 1322-1323 (1984).

${ }^{37}$ B.J. Skromme, S.S Bose, B. Lee, T.S. Low, T.R. Lepkowski, R.Y. DeJule, G.E. Stillman and J.C.M. Hwang, Appl. Phys. Lett. 58, 4685-4702 (1985).

${ }^{38}$ E.C. Larkins, E.S. Hellman, D.G. Schlom, J.J. Harris, M.H. Kim and G.E. Stillman, J. Cryst. Growth 81, 344-348 (1987).

${ }^{39}$ C.H. Park and D.J. Chadi, Phys. Rev. B 54, 14246-14249 (1996).

${ }^{40}$ M.-H. Du and S.B. Zhang, Phys. Rev. B 72, 075210 (2005).

${ }^{41}$ C. Giannini, C. Gerardi, L. Tapfer, A. Fischer and K.H. Ploog, 
J. Appl. Phys. 74, 77-81 (1993).

${ }^{42}$ R. Fletcher, E. Zaremba, M. D'Iorio, C.T. Foxon and J.J. Harris, Phys. Rev. B 41, 10649-10666 (1990).

${ }^{43}$ C. Gerl, J. Bauer and W. Wegscheider, J. Cryst. Growth 301302, 145-147 (2007).

${ }^{44}$ H. Ito, O, Nakajima and T. Ishibashi, Appl. Phys. Lett. 62, 62, 2099-2101 (1993).

${ }^{45}$ R.F. Pierret, Advanced Semiconductor Fundamentals; Prentice Hall, (2002).

${ }^{46}$ E.F. Schubert, L.N. Pfeiffer, K.W. West, H.S. Luftman and G.J. Zydzik, Appl. Phys. Lett. 64, 22380-2240 (1994).

${ }^{47}$ S. Schuppler, D.L. Adler, L.N. Pfeiffer, K.W. West, E.E Chaban and P.H. Citrin, Appl. Phys. Lett. 63, 2357-2359 (1993).

${ }^{48}$ C. Domke, Ph. Ebert, M. Heinrich and K. Urban, Phys. Rev. B
54, 10288-10291 (1996).

${ }^{49}$ S.J. MacLeod, K. Chan, T.P. Martin, A.R. Hamilton, A. See, A.P. Micolich, M. Aagesen and P.E. Lindelof, Phys. Rev. B 80, 035310 (2009).

${ }^{50}$ M. Heiblum, J. Vac. Sci. Technol. B 3, 820-822 (1985).

${ }^{51}$ L.N. Pfeiffer, E.F. Schubert, K.W. West and C.W. Magee, Appl. Phys. Lett. 58, 2258-2260 (1991)

${ }^{52}$ W.R. Clarke, A.P. Micolich, A.R. Hamilton, M.Y. Simmons, K. Muraki and Y. Hirayama, J. Appl. Phys., 99, 023707 (2006).

${ }^{53}$ B. Laikhtman, M. Heiblum and U. Meirav, Appl. Phys. Lett. 57, 1557-1559 (1990).

${ }^{54}$ N.M. Cho, D.J. Kim, A. Madhukar, P.G. Newman, D.D. Smith, T. Aucoin and G.J. Iafrate, Appl. Phys. Lett. 52, 2037-2039 (1988).

${ }^{55}$ D.V. Lang, J. Appl. Phys. 45, 3023-3032 (1974). 